Case Report

\title{
Bradycardia Leading to Asystole Following Dexmedetomidine Infusion during Cataract Surgery: Dexmedetomidine-Induced Asystole for Cataract Surgery
}

\author{
Amaniti Aikaterini (D), Dalakakis Ioannis, ${ }^{1}$ Gkinas Dimitrios, ${ }^{1}$ Sapalidis Konstantinos, \\ Grosomanidis Vasilios, ${ }^{1}$ and Papazisis George ${ }^{2}$
}

${ }^{1}$ AHEPA University Hospital, Aristotle University of Thessaloniki, Thessaloniki, Greece

${ }^{2}$ Laboratory of Pharmacology, Aristotle University of Thessaloniki, Thessaloniki, Greece

Correspondence should be addressed to Amaniti Aikaterini; amanitik@gmail.com

Received 17 August 2018; Revised 8 November 2018; Accepted 25 November 2018; Published 5 December 2018

Academic Editor: Neerja Bhardwaj

Copyright (C) 2018 Amaniti Aikaterini et al. This is an open access article distributed under the Creative Commons Attribution License, which permits unrestricted use, distribution, and reproduction in any medium, provided the original work is properly cited.

\begin{abstract}
Dexmedetomidine is an elective alpha-2 adrenergic agonist, being used in anesthesia practice. It offers sedative, anxiolytic, analgesic, muscle relaxant, and sympathetic lytic properties. While respiratory effects are considered minor, there are important cardiovascular side effects with bradycardia and hypotension being the most common. This article presents a case of a patient, with significant comorbidities and polypharmacy, who was scheduled for cataract surgery under dexmedetomidine sedation. During the administration, severe hemodynamic deterioration was observed with bradycardia and hypotension leading to asystole. Cardiac arrest was successfully managed in accordance with the guidelines of the European Resuscitation Council. Despite the promising role of dexmedetomidine in anesthesia practice, the associated adverse effects cannot be ignored. For this reason it is obligatory to have the minimum mandatory monitoring in accordance with the safety and quality guidelines.
\end{abstract}

\section{Introduction}

Dexmedetomidine is unique for its sedative and anxiolytic properties in the operating room or intensive care unit, due to its minimal respiratory effects [1]. Dexmedetomidineinduced cardiovascular effects are usually characterized by lowered heart rate and blood pressure [2,3]. While cases of bradycardia or asystole under dexmedetomidine have been documented [4], asystole has been perpetuated by either the coexistence of factors like spinal anesthesia [4] or increased doses [5]. Moreover, little is known about the interaction of dexmedetomidine with other medications. We describe a case of bradycardia and asystole, following dexmedetomidine infusion, in a patient undergoing cataract surgery.

\section{Case Report}

A male patient, 54 years old, $170 \mathrm{~cm}, 80 \mathrm{~kg}$, American Society of Anesthesiologist Physical Status (ASA-PS) graded 3, was planned for elective cataract surgery. Previous medical history of the patient revealed severe psychotic disorder and drug-resistant epilepsy, diagnosed by the age of 6 . He also suffered from congenital bilateral nerve palsy and stinging, type II diabetes mellitus, and dyslipidemia. Smoking, alcohol abuse, or allergies were not reported. Patient's activity, estimated by metabolic equivalents (METS), could not be assessed. His current medication included Levetiracetam mg $1500 \mathrm{mg}$ twice daily, Oxcarbazepine $400 \mathrm{mg}$ twice daily, Clobazam $10 \mathrm{mg}$ once daily, Ebastine $20 \mathrm{mg}$ every other day, Pregabalin mg $150 \mathrm{mg}$ three times daily, Risperidone $2 \mathrm{mg}$ twice daily, Metformin $500 \mathrm{mg}$ twice daily, Ezetimibe $10 \mathrm{mg}$ once daily, Eicosapentaenoic acid $1000 \mathrm{mg}$ once daily, and Omeprazole $20 \mathrm{mg}$ once daily.

Due to poor communication and cooperation, the ophthalmologist in charge decided to operate the patient under general anesthesia. During preoperative screening, clinical examination did not reveal pathological findings, ECG was reported without specific lesions, and chest X-ray was 
normal. Preoperative airway evaluation revealed prognostic factors of difficult airway: Mallampati classification grade III, thyromental distance (TMD) $<6 \mathrm{~cm}$, median teeth distance $<3 \mathrm{~cm}$, and moderate cervical spine mobility. Due to anticipated difficult intubation and based on the poor physical status, a sedation technique with dexmedetomidine was decided instead of general anesthesia.

After standard monitoring and intravenous line establishment, the patient was prehydrated with $300 \mathrm{ml}$ of Ringer's Lactate, followed by a single bolus of $50 \mathrm{mcg}$ of Fentanyl as an adjuvant analgesic.

Infusion of dexmedetomidine started at a loading dose of $1 \mathrm{mcg} / \mathrm{kg}$ for 10 minutes, followed by a maintenance dose of $0.4 \mathrm{mcg} / \mathrm{kg} / \mathrm{h}$. However, after 17 minutes of commencement of dexmedetomidine infusion and before surgery, the patient suddenly suffered bradycardia with hemodynamic collapse. Blood pressure was $75 / 45 \mathrm{mmHg}$ and heart rate $40 \mathrm{bpm}$. Immediately $0.5 \mathrm{mg}$ of atropine was administered and infusion of dexmedetomidine stopped at the same time. Seconds after, cardiac arrest with asystole occurred. Advanced life support and cardiopulmonary resuscitation was implemented according to the European Resuscitation Council algorithm. Return of spontaneous circulation (ROSC) occurred at about 4 minutes after 2 cycles of cardiopulmonary resuscitation. The operation was postponed. When the patient gained satisfactory neurological status of consciousness, he was transferred to the Coronary Unit for further intensive care. Few hours later he was transferred to the ward in good overall condition.

\section{Discussion}

Bradycardia and asystole after dexmedetomidine infusion is a well-described phenomenon [4]. Some of them have been attributed to the administered doses or surgical stimulation, while other authors consider cardiac morbidity as a cofactor.

In our case, dexmedetomidine was administered at a dose of $1 \mathrm{mcg} / \mathrm{kg}$ for 10 minutes followed by continuous infusion at a rate of $0.4 \mu \mathrm{g} / \mathrm{kg} / \mathrm{h}$. Based on a model of simulated concentration, a concentration of $0.62 \mathrm{ng} / \mathrm{ml}$ was estimated for our patient [4]. Depression of cardiac function would have been induced when the plasma concentration of dexmedetomidine exceeded $1.2 \mathrm{ng} / \mathrm{ml}$ [5] although plasma concentration of dexmedetomidine was not measured in this case. Takada et al. concluded that risk of cardiac arrest is not necessarily associated with the blood concentration of dexmedetomidine [4].

It is worthwhile to note that our concerns were about polypharmacy and the potential interactions between the patient's current medication and dexmedetomidine. There is no data in the literature regarding the coadministration of dexmedetomidine with many other drugs. However, the combination of dexmedetomidine with benzodiazepines has been used for the treatment of alcohol withdrawal syndrome. Double blind randomized trials demonstrated a statistically better outcome in the group receiving dexmedetomidine and benzodiazepines compared to the benzodiazepine monotherapy group. Nevertheless, bradycardia was reported as a common event with dexmedetomidine [6].
Another key point is the possible interaction between dexmedetomidine and pregabalin. While there are no reports regarding their coadministration, pregabalin has been found to lower heart rate and attenuate sympathetic outflow during laryngoscopy [7].

The introduction of dexmedetomidine offers the anesthetist many advantages without causing respiratory depression. But it does not lack other side effects with bradycardia being the prominent one. For this reason, it is mandatory to have a standard monitoring as well as the alertness to manage critical incidents.

\section{Conflicts of Interest}

The authors declare that they have no conflicts of interest.

\section{Acknowledgments}

This was supported by the Department of Anesthesia and Critical Care, AHEPA University Hospital, Aristotle University of Thessaloniki, Thessaloniki, Greece.

\section{References}

[1] G. M. Keating, "Dexmedetomidine: a review of its use for sedation in the intensive care setting," Drugs, vol. 75, no. 10, pp. 1119-1130, 2015.

[2] G. Piao and J. Wu, "Systematic assessment of dexmedetomidine as an anesthetic agent: A meta-analysis of randomized controlled trials," Archives of Medical Science, vol. 10, no. 1, pp. 1924, 2014.

[3] Y. Nagasaka, A. Machino, K. Fujikake, E. Kawamoto, and M. Wakamatsu, "Cardiac arrest induced by dexmedetomidine," The Japanese Journal of Anesthesiology, vol. 58, no. 8, pp. 987-989, 2009.

[4] K. Takata, Y. U. Adachi, K. Suzuki, Y. Obata, S. Sato, and K. Nishiwaki, "Dexmedetomidine-induced atrioventricular block followed by cardiac arrest during atrial pacing: A case report and review of the literature," Journal of Anesthesia \& Clinical Research, vol. 28, no. 1, pp. 116-120, 2014.

[5] T. J. Ebert, J. E. Hall, J. A. Barney, T. D. Uhrich, and M. D. Colinco, "The effects of increasing plasma concentrations of dexmedetomidine in humans," Anesthesiology, vol. 93, no. 2, pp. 382-394, 2000.

[6] K. Bielka, I. Kuchyn, and F. Glumcher, "Addition of dexmedetomidine to benzodiazepines for patients with alcohol withdrawal syndrome in the intensive care unit: a randomized controlled study," Annals of Intensive Care, vol. 5, no. 1, p. 33, 2015.

[7] S. Parveen, D. S. Negi, R. Kumar, and M. C. Bagwan, "Oral clonidine vs oral pregabalin premedication to attenuate pressor response to direct laryngoscopy in patients undergoing laparoscopic cholecystectomy: A randomized double blind study," Journal of Clinical and Diagnostic Research, vol. 10, no. 9, pp. UC21-UC25, 2016. 


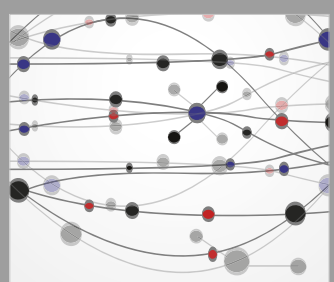

The Scientific World Journal
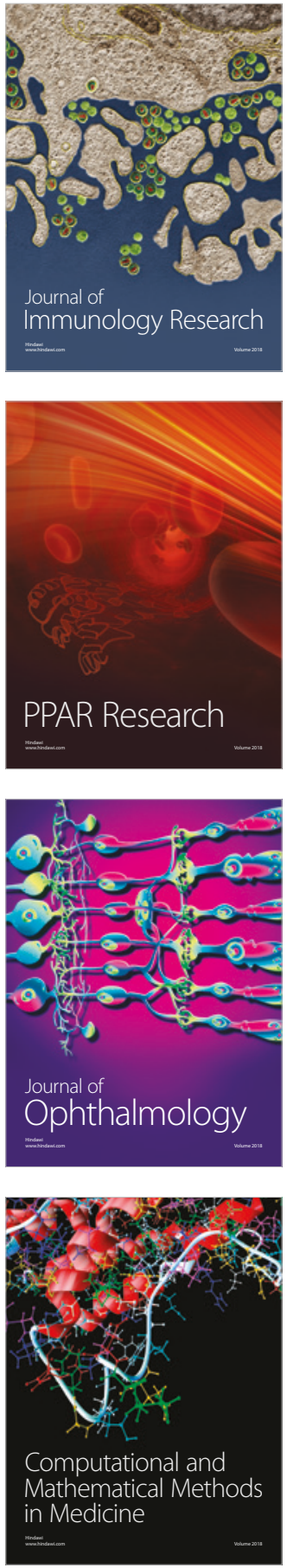

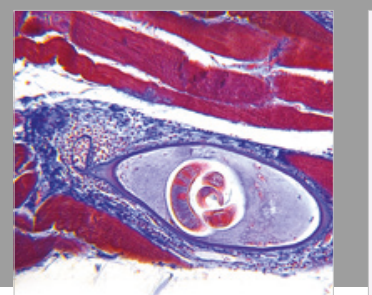

Gastroenterology Research and Practice

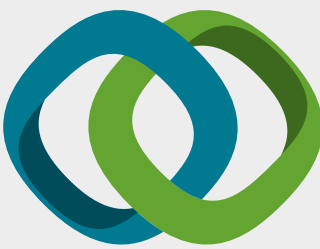

\section{Hindawi}

Submit your manuscripts at

www.hindawi.com
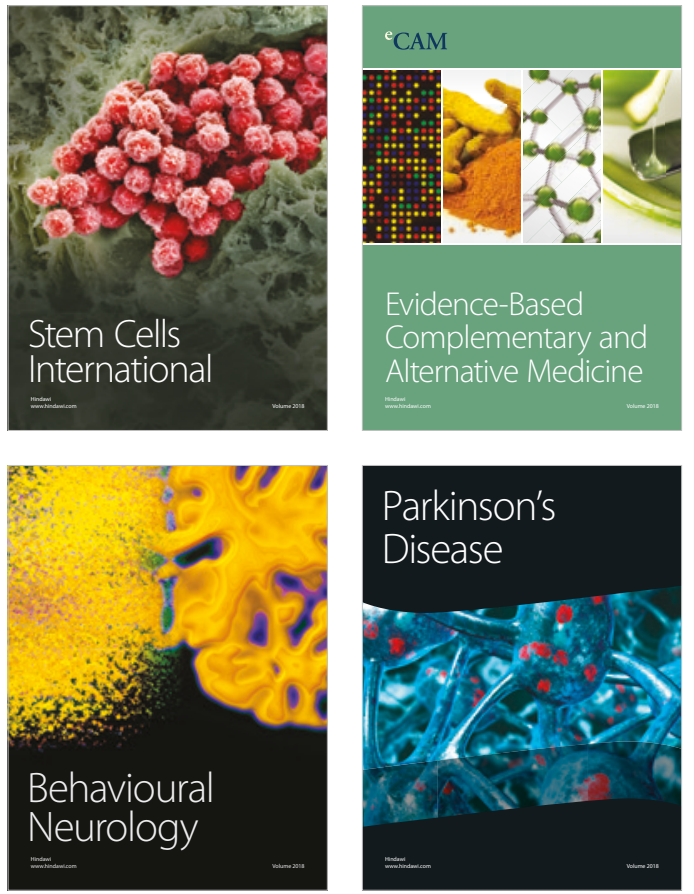

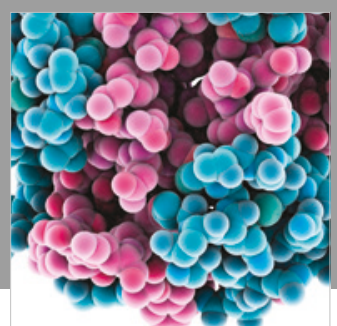

ournal of

Diabetes Research

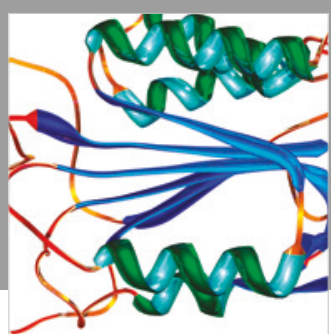

Disease Markers
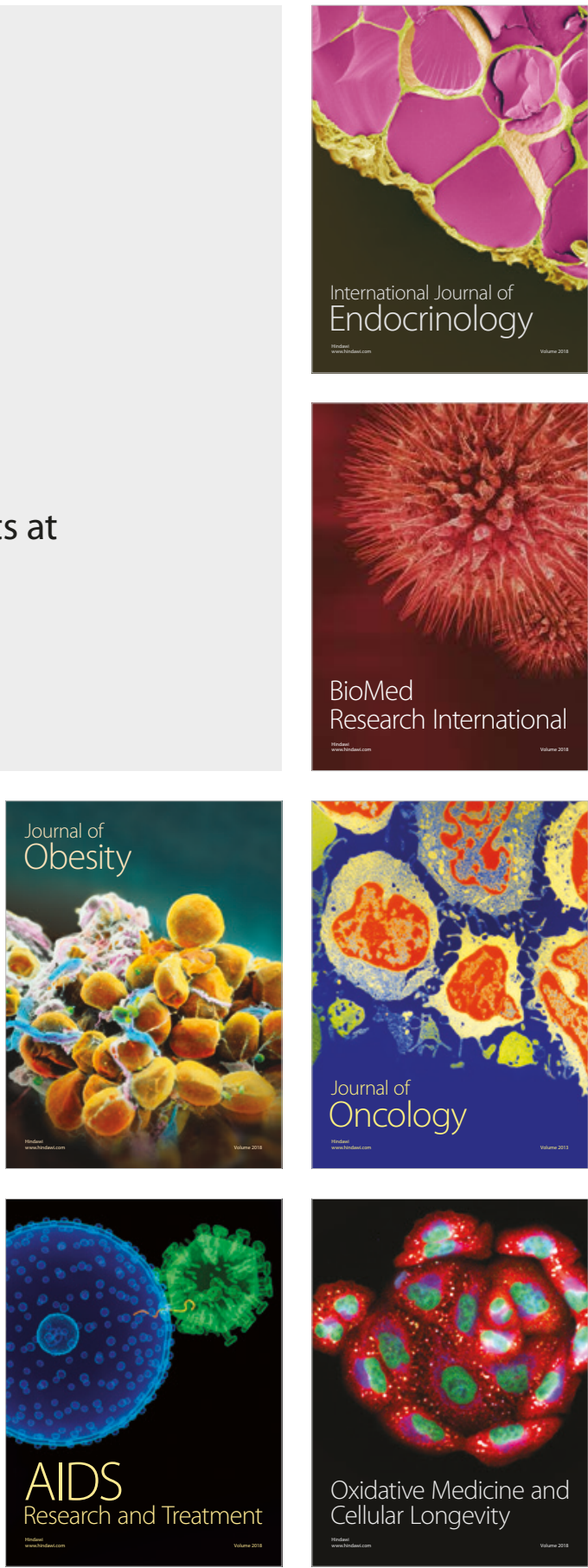